\title{
Facial nerve paralysis as the first manifestation of tetanus in a 4-year-old girl
}

\author{
Arumugam Jayavardhana ${ }^{1}$, A Vijayalakshmi ${ }^{2}$ \\ Sri Lanka Journal of Child Health, 2011; 40(3): 136-137
}

DOI: $\underline{\text { http://dx.doi.org/10.4038/sljch.v40i3. } 3515}$

\begin{abstract}
We report a case of a 4-year-old girl who developed right sided facial palsy following injury to forehead. Two days later, she developed trismus which gradually progressed to generalized tetanus. A literature search shows that this child is the first to present with facial palsy as the initial manifestation of tetanus in Indian literature.
\end{abstract}

(Key words: Tetanus; facial palsy; cephalic tetanus)

\section{Introduction}

Cephalic tetanus is a rare form of tetanus defined as trismus plus paralysis of one or more cranial nerves. The seventh cranial nerve is most commonly involved. We report a 4-year-old girl who had facial palsy as the initial manifestation of tetanus.

\section{Case report}

A 4-year-old girl presented with a history of right sided facial palsy for 4 days and an inability to open the mouth for 2 days. She sustained an injury over the forehead on the right side 6 days ago. She developed weakness of the right side of face two days after injury for which she was taken to a neurologist who diagnosed right sided Bell's palsy and started the child on oral B-complex vitamins. She was immunized with a single dose of DPT only, at two months of age. On examination, she was afebrile and had a wound measuring $1 \mathrm{~cm}$ on the forehead. She had right sided lower motor neuron facial palsy (Figure 1) and normal sensorium. She had trismus and dysphagia. The findings of the remainder of the central nervous system, the cardiovascular system and respiratory system were normal. A diagnosis of

\section{${ }^{1}$ Asst. Professor of Paediatrics, ${ }^{2}$ Professor of Paediatrics, Postgraduate Institute of Medical Sciences and Research, Peelamedu, Coimbatore, Tamil Nadu, India}

(Received on 02 September 2010: Accepted on 29 October 2010) tetanus was made based on the clinical findings. She was started on tetanus immunoglobulin, intravenous diazepam and crystalline penicillin. The wound on the forehead was cleaned daily and betadine ointment applied. She developed generalized tetanic spasms with ophisthotonus from the third hospital day (Figure 2). She improved gradually with the above treatment and supportive care and recovered fully over a period of 3 weeks. Her right sided facial palsy had recovered completely at the time of discharge.

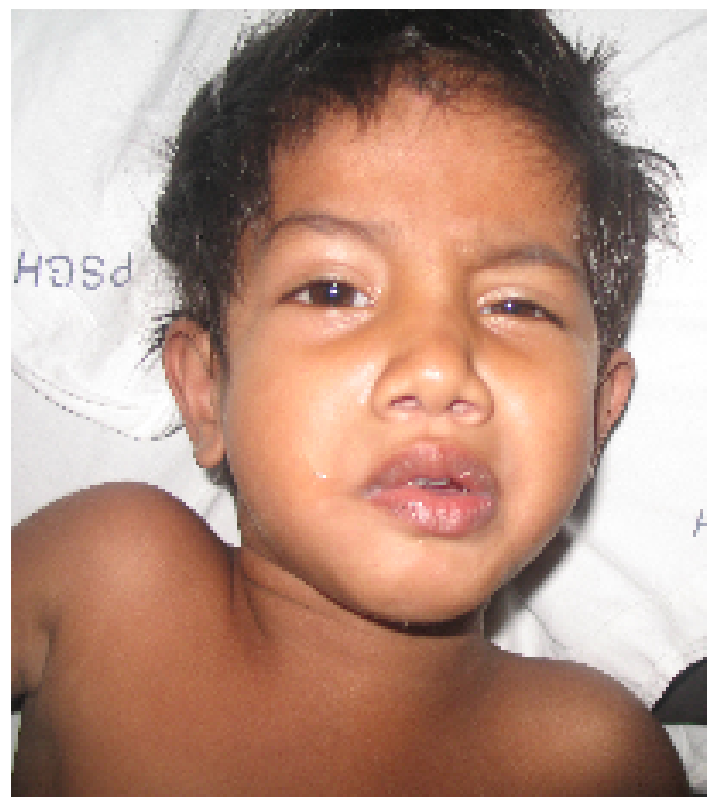

Figure 1: Photograph showing right facial palsy

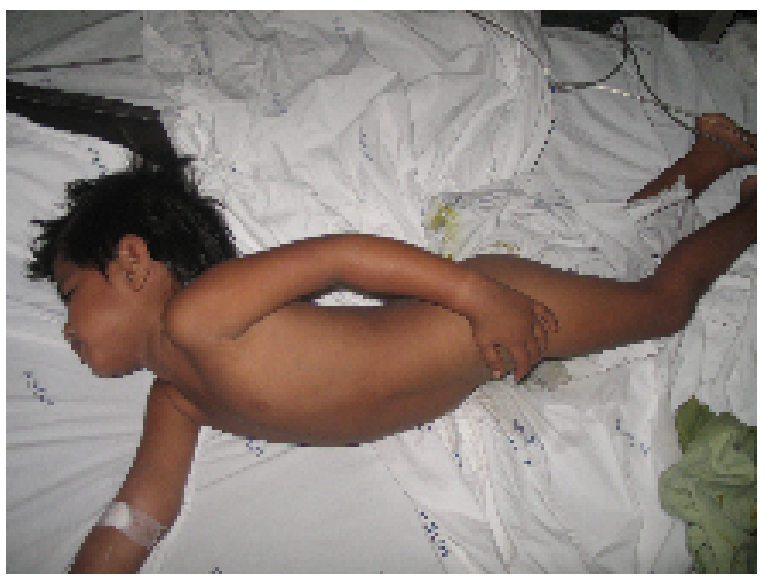

Figure 2: Photograph showing ophisthotonus 


\section{Discussion}

Although safe and effective vaccines are available to protect against tetanus, cases of tetanus among children continue to occur. In a small proportion of cases of tetanus cranial nerve palsy is apparent either as a presenting sign or developing in the course of the disease. This variant has been called cephalic tetanus ${ }^{1}$. The incidence of cephalic tetanus ranges from 0.9 to $3 \%^{2}$. Among the cranial nerves, seventh cranial nerve is most commonly involved ${ }^{1}$. Facial nerve palsy preceding the onset of trismus is very rare and it has been reported in a few adult patients ${ }^{3-7}$. This is the first and youngest child who presented with facial palsy preceding trismus in the Indian literature. Cases of tetanus where seventh cranial nerve palsy precedes trismus may be misdiagnosed as Bell's palsy as happened in our case.

Two third of cephalic tetanus cases may progress to generalized tetanus ${ }^{2}$. Mortality in cephalic tetanus and ordinary tetanus is determined by the severity of the generalized disease with little prognostic significance attached to cranial nerve palsy ${ }^{1}$. Recovery of cranial nerve palsy is complete if the patient survives the illness. Site of lesion and nature of pathology in cranial nerve palsies associated with tetanus are still not established. Cranial nerve palsy may be due to nuclear rather than peripheral lesion ${ }^{1}$. This case suggests that when a child presents with facial palsy following trauma to face, the physician should consider the possibility of tetanus in the differential diagnosis.

\section{References}

1. Park DM. Cranial nerve palsies in tetanus: Cephalic tetanus. J Neurol Neurosurg Psychiat $1970 ; 33: 212-5$. http://dx.doi.org/10.1136/jnnp.33.2.212

2. Jagoda A, Riggio S, Burguieres T. Cephalic tetanus: a case report and review of literature. Am J Emergency Med 1988 ; 6 : 128-30. http://dx.doi.org/10.1016/0735-6757(88)90049-6

3. Schon F, O'Dowd L, White J, Begg N. Tetanus: delay in diagnosis in England and Wales. $J$ Neurol Neurosurg Psychiat 1994; 57:1006-7. http://dx.doi.org/10.1136/jnnp.57.8.1006

4. Chocarro Martinez A, Hernandez Salvador J, Palomar LM, Gonzalez - Lopez A, De Diego Gomez JM. Facial parálysis as first manifestation of tetanus. Ann Med Intern 1995; 12: 51 .

5. Tonduangu KD, Jungfer F, Nahila M, Ranarivelo L, Mogylevsky A, Gaillard C. et al. Cephalic tetanus and Bell's palsy in an elderly man. Ann Med Intern (Paris) 2003; 154: 190 -1.

6. Searl S S. Minor trauma, disastrous results. Surv Ophthalmol 1987; 31: 337-42. http://dx.doi.org/10.1016/0039-6257(87)90118-4

7. Agarwal A K, Mudgerikar S, Sangla K S, Gogna A, Jain A. Facial palsy of uncommon aetiology (cephalic tetanus). $J$ Assoc Physicians India 1995; 43: 229 - 30. 